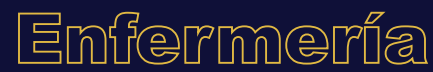

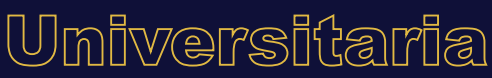

\section{Perspectiva de mujeres mexicanas sobre lactancia materna asociada al cambio del modelo cultural}

\section{Mexican women breastfeeding perspective associated with the cultural model change}

\section{Perspectiva das mulheres mexicanas sobre a aleitamento materno associado ao câmbio do modelo cultural}

\author{
A. Torres-Montalvo ${ }^{\mathrm{a},{ }^{*}}$, J.E. Suárez-Conejero ${ }^{\mathrm{b}, 2}$, \\ M.R. Cerros-Aristorena ${ }^{\mathrm{c}, 3}$ \\ ORCID \\ a $0000-0002-5882-8092$ \\ b $0000-0002-5376-5950$ \\ c 0000-0003-0894-4113 \\ ${ }^{1}$ Universidad Nacional Autónoma de México, Escuela Nacional de Enfermería y Obstetricia, \\ Ciudad de México, México \\ ${ }^{2}$ Universidad Nacional Autónoma de México, Escuela Nacional de Trabajo Social, Ciudad de \\ México, México \\ ${ }^{3}$ Universidad Autónoma de Zacatecas, Unidad Académica de Enfermería, Zacatecas, México \\ Recibido: 09 octubre 2019 \\ Aceptado: 16 enero 2020
}

RESUMEN

Introducción: La lactancia materna es importante para el desarrollo saludable del recién nacido. A pesar de sus beneficios, la decisión de no amamantar se incrementa entre las mujeres de la Ciudad de México debido a la influencia de la globalización y del cambio de modelo cultural moderno al posmoderno.

*Autora de correspondencia. Correo electrónico: masteragri@comunidad.unam.mx https://doi.org/10.22201/eneo.23958421e.2020.2.767

1665-7063/@ 2020 Universidad Nacional Autónoma de México, Escuela Nacional de Enfermería y Obstetricia. Este es un artículo Open Access bajo la licencia CC BY-NC-ND (http://creativecommons.org/licenses/by-nc-nd/4.o/). 
Objetivo: Comprender la perspectiva de las mujeres mexicanas sobre el ofrecer y abandonar la lactancia materna asociada al cambio del modelo cultural.

Metodología: Se llevó a cabo un estudio cualitativo con enfoque exploratorio, descriptivo: participaron 12 diadas (madre-hijo lactante), residentes de la Ciudad de México, quienes ofrecieron y abandonaron la lactancia materna. Se realizaron entrevistas en profundidad. Los datos obtenidos se grabaron, transcribieron y codificaron para garantizar el rigor de la investigación.

Resultados: Se reconocieron dos categorías que sustentan el por qué las mujeres brindan o abandonan la lactancia materna, vistas desde el modelo cultural. En el modelo moderno las mujeres consideran una convicción sentida el ofrecer lactancia materna, reconocen los beneficios de dicho alimento para su hijo; mientras que, en el modelo posmoderno, las mujeres tienen una convicción negativa de amamantar.

Conclusiones: Al asociar la lactancia materna con modelos culturales, en el moderno se considera la lactancia como un deber, mientras que en el posmoderno un derecho. Al encontrarnos en transición entre ambos modelos, las mujeres están influenciadas por discursos y prácticas culturales que en ocasiones son contradictorias. Comprender estos fenómenos permitirá diseñar estrategias efectivas desde la enfermería para fomentar la lactancia materna.

Palabras clave: Lactancia materna; lactancia materna exclusiva; cambio cultural; toma de decisiones; México.

\section{ABSTRACT}

Introduction: Breastfeeding is an important activity for the healthy development of the newborn, but nevertheless the benefits, the decision of women to avoid breastfeeding is becoming more prevalent in Mexico City. Perhaps this decision is the result of the influence of the globalization process with its cultural model change from the modern to the postmodern.

Objective: To better understand the perspective of Mexican women regarding breastfeeding or not breastfeeding and the relationship of this decision with the cultural model change.

Methodology: This is a qualitative, descriptive, and exploratory study on 12 mothers in Mexico City, who decided to terminate breastfeeding their babies. In-Depth interviews were conducted. The gathered data were recorded, transcribed, and coded in order to procure rigour of research.

Results: Two categories related to the cultural model that reflect why women breastfeed or not were identified. In the modern model, women consider breastfeeding as a solid conviction which has benefits to their babies, while in the postmodern model, women tend to have a negative image of breastfeeding.

Conclusions: From the association of breastfeeding with the cultural models, in the modern model, women consider breastfeeding as a duty, while in the postmodern one, women consider breastfeeding as a right. Currently, we are in a transition between these possibly contradicting cultural models. Therefore, better understanding these phenomena can help nursing professionals design strategies which can foster a healthy decision on breastfeeding. Keywords: Breast feeding; exclusive breast feeding; cultural change; decision making; Mexico. 


\section{RESUMO}

Introdução: O aleitamento materno é importante para o desenvolvimento saudável do recém-nascido. Apesar de seus benefícios, a decisão de não amamentar aumenta entre as mulheres da Cidade do México devido à influência da globalização e da mudança do modelo cultural moderno para o pós-moderno.

Objetivo: Compreender a perspectiva das mulheres mexicanas sobre a oferta e o abandono do aleitamento materno associado à mudança do modelo cultural.

Metodologia: Realizou-se um estudo qualitativo com abordagem exploratória e descritiva: participaram 12 díades (mãe-filho em lactação), residentes na Cidade do México, que ofereceram e abandonaram a amamentação. Entrevistas em profundidade foram realizadas. Os dados obtidos foram gravados, transcritos e codificados para garantir o rigor da pesquisa. Resultados: Foram reconhecidas duas categorias que sustentam porque as mulheres oferecem ou abandonam a amamentação, visto a partir do modelo cultural. No modelo moderno, as mulheres consideram o fato da amamentação uma convicção sincera, reconhecem os benefícios dessa alimentação para o filho; enquanto no modelo pós-moderno as mulheres têm uma convicção negativa de amamentar.

Conclusões: Ao associar o aleitamento materno aos modelos culturais, no moderno amamentar é considerado um dever, enquanto na pós-moderna um direito. Ao encontrarmos em transição entre os dois modelos, as mulheres são influenciadas por discursos e práticas culturais que às vezes são contraditórios. Compreender esses fenômenos permitirá o desenho de estratégias de enfermagem eficazes para promover o aleitamento materno.

Palavras chave: Aleitamento materno; aleitamento materno exclusivo; câmbio cultural; tomada de decisões; México.

\section{INTRODUCCIÓN}

La leche materna es el alimento ideal para el recién nacido; pues satisface tanto las necesidades inmunológicas como nutricionales para un buen crecimiento y desarrollo. La Organización Mundial de la Salud (OMS) y el Fondo de las Naciones Unidas para la Infancia (UNICEF) recomiendan la lactancia materna exclusiva (LME) durante los primeros seis meses de vida, posteriormente la introducción de alimentos apropiados y seguros de acuerdo a la edad, así como el mantenimiento de la lactancia hasta los 2 años o más.

La OMS define la LME como la única forma de alimentación que obtiene el niño del pecho de la madre o de una nodriza sin recibir ningún tipo de alimentos líquidos o sólidos, ni siquiera agua (con la excepción de solución de rehidratación oral, gotas o jarabes de suplementos vitamínicos, minerales o medicamentos).

La Lactancia Materna (LM) responde a un hecho biológico; sin embargo, está sujeta a influencias económicas, culturales, así como sociales². Durante la última década ha perdido importancia debido a la comercialización y a la presencia de diversos productos lácteos artificiales, aunado a la incorporación de las mujeres al mercado laboral (formado en su mayoría por mujeres en edad reproductiva) $)^{3}$, lo que influye en la decisión de las madres lactantes al deteste prematuro, generando un impacto negativo en la salud de los niños. Como consecuencia de ello, las enfermedades crónicas del lactante van en aumento las cuales constituyen las principales causas de morbilidad y mortalidad en el país4. 
Algunos estudios 5 señalan diversas ventajas de la LM para los lactantes, madres, familia y sociedad, las cuales incluyen beneficios para la salud, estado nutricional, respuesta inmunológica, crecimiento y desarrollo, así como aspectos psicológicos, sociales, económicos y ambientales.

No obstante, a pesar de los beneficios indiscutibles que aporta la LM y los esfuerzos de diversos organismos nacionales e internacionales para destacar la importancia de dicha práctica, a nivel mundial se estima que solamente el 34.8\% de lactantes reciben LME durante los primeros 6 meses de vida ${ }^{1}$, mientras que en la Región de Latinoamérica y el Caribe alrededor del $40 \%$ de los niños y niñas son amamantados ${ }^{6}$.

De acuerdo con los resultados de la Encuesta Nacional de Niños, Niñas y Mujeres (ENIM) de México 7 , la prevalencia de la LME durante los primeros 6 meses de vida de los bebés se duplicó en los últimos años, pasando de $14.4 \%$ en $2012^{8}$ a un $30.8 \%$ en $2015^{7}$. Aunque esto representa un importante avance, no es suficiente para cumplir con el derecho de todos los niños y niñas a una buena nutrición desde su nacimiento. Los grupos más afectados son los niños que viven en zonas rurales, donde se identifica un descenso considerable de la LM (de 36.9 a 18.5\%). En general, el medio rural ha sido el más afectado, sobre todo la región sur del país que presenta el tercil más bajo de nivel socioeconómico9 ${ }^{9}$. Las causas asociadas al descenso de la práctica de la LME resultan multifactoriales (físicas, estructurales y socioculturales). Cabe señalar que para esta investigación el aspecto sociocultural es el de mayor relevancia debido al enfoque y relación con el cambio de modelo cultural.

El cambio de modelo cultural (CMC) se entiende como un conjunto de principios éticos y morales que van más allá de los valores o las creencias, se trata de algo que define, determina, orienta, condiciona las representaciones sociales y normas de comportamiento que sirven para dar sentido y legitimar las prácticas en todos los campos de las relaciones sociales.

Según el sociólogo Guy Bajoit este cambio se caracteriza por la pérdida de credibilidad de un modelo cultural de la primera modernidad (realista) a un modelo cultural de la segunda modernidad (subjetivista) ${ }^{10}$. Para esta investigación se hace alusión de estos términos como modelo cultural moderno (MCM) y la sustitución gradual por otro nuevo, que puede llamarse modelo cultural posmoderno (MCP).

Para comprender mejor los términos se entiende que el MCM se basaba en la creencia, la razón, así como el deber, los cuales orientan y dan un sentido a las conductas de las personas antiguas, mientras que el MCP es el que apela a la persona como sujeto autónomo y responsable con respecto a sí mismo. Los individuos actuales critican los sentidos culturales antiguos, existe un cambio en todos los campos relacionales de la vida social, donde las prácticas se transforman considerablemente ${ }^{11}$.

Referente a lo anterior, queda de manifiesto la transición de un MCM basado en los deberes a un MCP centrado en los derechos; el cual afecta de manera directa el abandono de la LME. Cabe mencionar que el cambio de modelos no se produce de forma rápida, sino que se genera paulatinamente.

De acuerdo con la revisión de la literatura nacional e internacional, existen pocos estudios desde esta perspectiva y metodología (cualitativa) que revelen motivos del porqué las mujeres mexicanas deciden mantener o abandonar la LME visto desde el modelo cultural, situación que dificulta comprender y contrarrestar de manera eficiente el bajo índice de la LME.

La incidencia del abandono de la LME es variable. En el 2015 Xavier-Amaral et al. ${ }^{12}$ encontraron que los principales factores que influyeron en las madres brasileñas para interrumpir la lactancia durante los primeros seis meses de vida del bebé fueron: poco conocimiento del vínculo madre-hijo, la reducción de los gastos familiares tras la llegada del niño, la hipogalactia, condiciones de salud 
del recién nacido y complicaciones mamarias. Por otra parte, en un estudio realizado en Canarias, Estévez-González et al. ${ }^{13}$ reportaron que las causas estaban relacionadas con dificultades durante la lactancia como poca leche y hambre del bebé, así como personales.

Con respecto en estudios realizados en México, diversos autores ${ }^{3,14,15}$ concordaron que los factores con mayor influencia para que ocurra el abandono precoz de la LME fueron: no tener leche, el niño no se llenaba, publicidad de alimentos infantiles, cambios de estilo de vida, roles asumidos por la mujer y falta de apoyo para desarrollar exitosamente la práctica del amamantamiento.

De acuerdo con estos resultados, es indudable que en nuestro país un gran número de mujeres inician la práctica de la lactancia materna; no obstante, en el transcurso de los meses la práctica va disminuyendo de una manera significativa, situación que nos aleja de las recomendaciones emitidas por la OMS. En general, los porcentajes de la práctica de la LM muestran un decremento en los últimos diez años según los datos emitidos por la Encuesta Nacional de Salud y Nutrición (ENSANUT), situación que preocupa a las instituciones de salud.

Conforme con lo expuesto anteriormente, se deduce que el tema del abandono de la LME es de gran interés a nivel internacional, ya que expone y devela importante evidencia acerca de los factores y beneficios que conlleva esta práctica, por tal razón, el objetivo principal de dicha investigación es comprender la perspectiva de las mujeres mexicanas sobre el ofrecer o abandonar la lactancia materna asociadas al CMC.

\section{METODOLOGÍA}

Estainvestigación se plantea desde un abordaje cualitativo ${ }^{16}$ bajo el marco metodológico exploratorioy descriptivo ${ }^{17}$.

La población de estudio se determinó por medio de características definidas con el objetivo de obtener las mejores respuestas. Mujer en edad reproductiva de 15 a 44 años con bebés menores de 2 años, miembros de un grupo de estimulación temprana pertenecientes a diversos estratos sociales (clase baja, media y alta) que ofrecieran o no LME, familiares de su entorno cercano (éste fue indicado por la madre) y residentes de la Ciudad de México. El número de las participantes se decidió por saturación, con la que se obtuvo una muestra de 12 mujeres.

La recolección de información se realizó entre enero y mayo del 2017 en dos fases; en la primera se localizó el centro de salud, contexto en donde se ubicaron a las participantes de diferentes estratos sociales, quienes asistían a un grupo de estimulación temprana; se gestionó la aprobación del Comité de Investigación de la institución para realizar el estudio, a las participantes se les explicó sobre el objetivo de la investigación y se les entregó el consentimiento informado. Como segunda fase se asistió a los hogares de las mujeres que decidieron participar para recabar información, con la finalidad de explorar los diferentes contextos en el que se encontraba la mujer lactante.

Los datos se recolectaron mediante entrevistas con ayuda de una guía estructurada, la cual consta de 4 preguntas ejes: indagar las causas del abandono o continuidad de la LME, identificar en qué modelo cultural se encuentra la madre, explorar el entorno familiar y examinar las características de la mujer lactante.

Se realizó prueba piloto de la guía de entrevista con dos mujeres que no asistían al grupo de estimulación temprana y que presentaban las mismas características de las participantes que se pretendía estudiar. Además, se validaron las preguntas y con los resultados se pudieron modificar algunas de estas para una mejor comprensión. 
En total se efectuaron 12 entrevistas con una duración aproximada de 50 minutos, de las que se obtuvieron criterios de saturación teórica. Todas se realizaron en ambientes controlados (hogar del informante), se grabaron en audio con el consentimiento de las participantes, a cada persona entrevistada se le asignó un pseudónimo con el objeto de resguardar su identidad.

Para el manejo de los datos se realizó la transcripción de la información con el programa Transana 3.10 Demonstration, mismo que nos permitía tener en la misma ventana acceso al audio y a la transcripción, así como una mejor organización y codificación de la información. Se consideró información suficiente mediante la saturación y la adecuación de los datos, se seleccionó la información según las necesidades teóricas del estudio.

En el análisis de la información se utilizó la propuesta operativa de Souza-Minayo et al. ${ }^{18}$ quienes proponen tipos temáticos en tres etapas; la primera corresponde al ordenamiento de los datos; la segunda a clasificación de los datos; la tercera al análisis final. Se inició con la transcripción de cada entrevista, se leyeron y volvieron a leer para reconocer los temas que más emanaban en los discursos; al final se asignaron etiquetas.

Los temas se agruparon por categorías y subcategorías según la frecuencia con la que se presentaban, los cuales fueron tomados como referencia para delinear las estructuras de relevancia en el discurso, lo que permitió reducir, categorizar, clarificar, sintetizar y comparar los testimonios.

Posteriormente, los datos se redujeron de manera conceptual a partir de una estructura sistemática para proponer categorías. Para finalizar, se realizó una interpretación global de los resultados de la investigación con el propósito de explicar las causas de abandono de la LME y asignación de zonas identitarias a las mujeres según el modelo cultural.

La presente investigación se realizó bajo los mandatos del Reglamento de la Ley General de Salud en Materia de Investigación para la Salud ${ }^{19}$, fue clasificada como investigación sin riesgo para la salud e integridad de las personas participantes. Asimismo, se consideraron los siguientes requerimientos: consentimiento informado, valoración riesgo-beneficio y selección de los sujetos de investigación conforme a la Comisión Nacional para la Protección de los Sujetos Humanos de Investigación Biomédica y del Comportamiento de los Estados Unidos de Norteamérica ${ }^{20}$.

Se apegó a lo establecido en la declaración de Helsinki ${ }^{21}$ al aplicar los principios bioéticos de no maleficencia, beneficencia y autonomía. También se utilizó el informe Belmont ${ }^{20}$ en la sección de principios éticos básicos para preservar la intimidad del informante, la confidencialidad, el anonimato, así como el respeto. El rigor científico estuvo dado por los principios de auditabilidad, credibilidad y la transferibilidad señalados por Guba et al. ${ }^{22}$

\section{RESULTADOS}

Tras el análisis de los datos se advirtió que las conductas sobre el seguir o abandonar la práctica de amamantamiento derivan una serie de causalidades, las cuales están relacionadas cultural y socialmente. Los discursos se agruparon por categorías según las áreas de indagación para determinar los significados más relevantes, de los cuales se descubrieron dos categorías claves y tres subcategorías.

Asimismo, se identificaron seis zonas identitarias para describir en cuál modelo cultural se encuentran las mujeres; pues debido a los cambios de modelos culturales, los individuos pueden adaptarse a uno de estos según lo que la sociedad les indique. Por esta razón Bajoit menciona que la estructura de la identidad individual comprende zonas articuladas entre sí23. 


\section{Categoría a. Convicción sentida de ofrecer Lactancia Materna Exclusiva}

Para las mujeres tener una convicción sentida de ofrecer LME se refleja en acciones que tienen que ver con los beneficios que brinda el dar este alimento natural tanto para la madre como para el bebé, así como el apoyo de la familia (Figura 1).

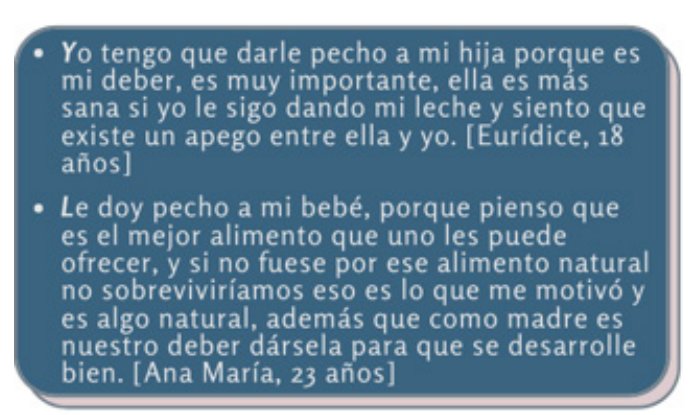

- Existe un apego de madre hija, tenemos un vínculo emocional y eso es bueno, para que sea más sana, nuestra leche es lo mejor. [Ana María, 23 años]

- Pienso que me alimento bien, mi niña tiene muchos beneficios con la lactancia materna, es el mejor alimento que le puedo dar. [Eurídice, 18 años]

- Tiene muchos nutrientes para mi bebé y se alimenta mejor, siempre he creído que es el mejor alimento que le puedo ofrecer. [Karina, 26 años]

- La lactancia materna es lo mejor para el bebé, los calostros lo protegen para que tenga más defensas y se enferme menos. [Yesica, 35 años]

- Mi esposo es el que siempre ha estado al pendiente de mí y de mi bebé, es mi apoyo incondicional y más cuando inicie con la lactancia, investigamos todos los beneficios que tenía al darle pecho y él me decía, tú dale lechita, porque es lo mejor. [Karina, 26 años]

- Mi mamá me dice que es muy bueno que yo le de la leche al niño, para que él crezca más sano y tenga protección para que no se enferme, todos piensan o mismo, porque cada vez que él toma yo voy produciendo más leche, pero sí todos me apoyan. [Yesica, 35 años]
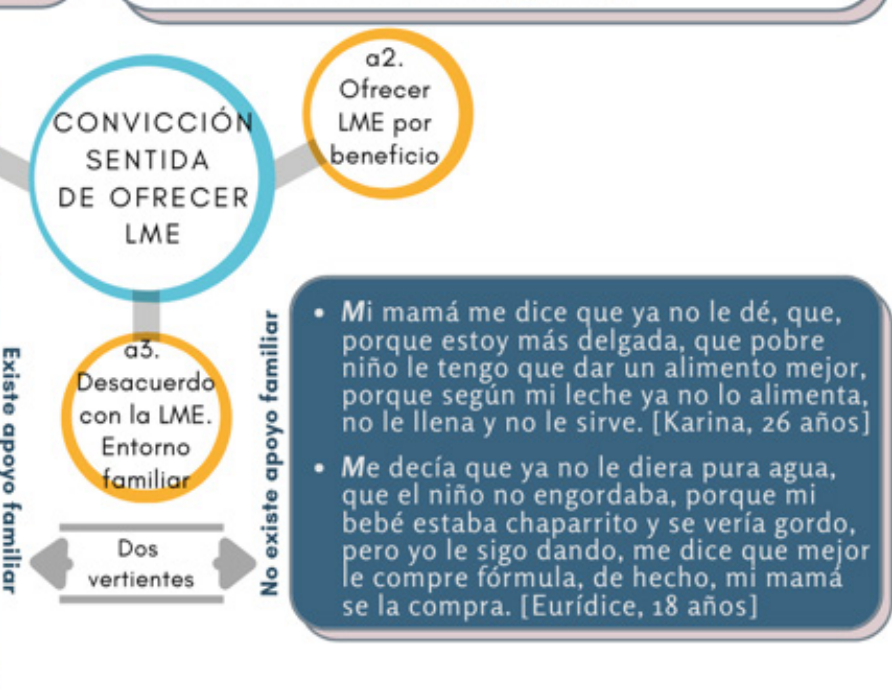

Figura 1. Esquematización sobre la opinión respecto a la categoría a. Convicción sentida de ofrecer LME

Subcategoría a1. Ofrecer LME por deber. Las razones culturales son las que sostienen el sentido del deber, estas se transmiten de generación en generación dentro del entorno familiar. Se trata de aquellas costumbres y manifestaciones que cada familia considera valiosas y las mantienen para que sean aprendidas por las nuevas generaciones como parte indispensable del legado cultural.

Subcategoría a2. Ofrecer LME por beneficio. La alimentación al seno materno es considerada como el mejor alimento que puede recibir el bebé, ya que esta regula su composición en función de sus necesidades, contiene los nutrientes más apropiados y necesarios en cada momento del desarrollo del niño.

Subcategoría a3. Desacuerdo de LME. Entorno familiar: La LM es un proceso biológico con una alta valoración cultural que tiene normas, creencias y prácticas diversas, las cuales al llevarlas a cabo reflejan las diferencias que existen en el comportamiento de las familias durante la crianza de sus hijos. En consecuencia, el entorno familiar se vuelve un factor crucial en la decisión de las madres, ya que influye positiva o negativamente en continuar o abandonar esta práctica. 
Por otra parte, dichos hallazgos coinciden con lo que plantea Bajoit en su discurso sobre el tránsito del modelo cultural basado en la razón hacia uno que se sustenta en la autorrealización autónoma ${ }^{24}$, es decir, dentro del MCM se encuentran las personas que poseen las mismas costumbres, idealismo, creencias y pensamientos, de modo que estas mujeres tienen el apoyo de su entorno (esposo, familia); pues no existen contradicciones entre ellos debido a que ambos se insertan en el mismo modelo. Por consiguiente, este tipo de mujeres se sienten autorrealizadas al ofrecer LME, disfrutan esta experiencia, además de la realización de sus deseos por la aprobación de los demás y por ende, están satisfechas de lo que son y de lo que hacen, razón por la cual se les da la siguiente asignación de zonas identitarias.

1. Mujer que tiene la convicción que debe dar LME y se encuentra en un entorno favorable a esta práctica; 2. Mujer que tiene la convicción que debe dar LME y la brinda a pesar de que se encuentra en un entorno vinculado al modelo posmoderno que la presiona para que no la brinde; 3. Mujer que tiene profunda convicción de no hacerlo, pero lo hace debido a la presión de su entorno vinculado al modelo moderno (Figura 2).

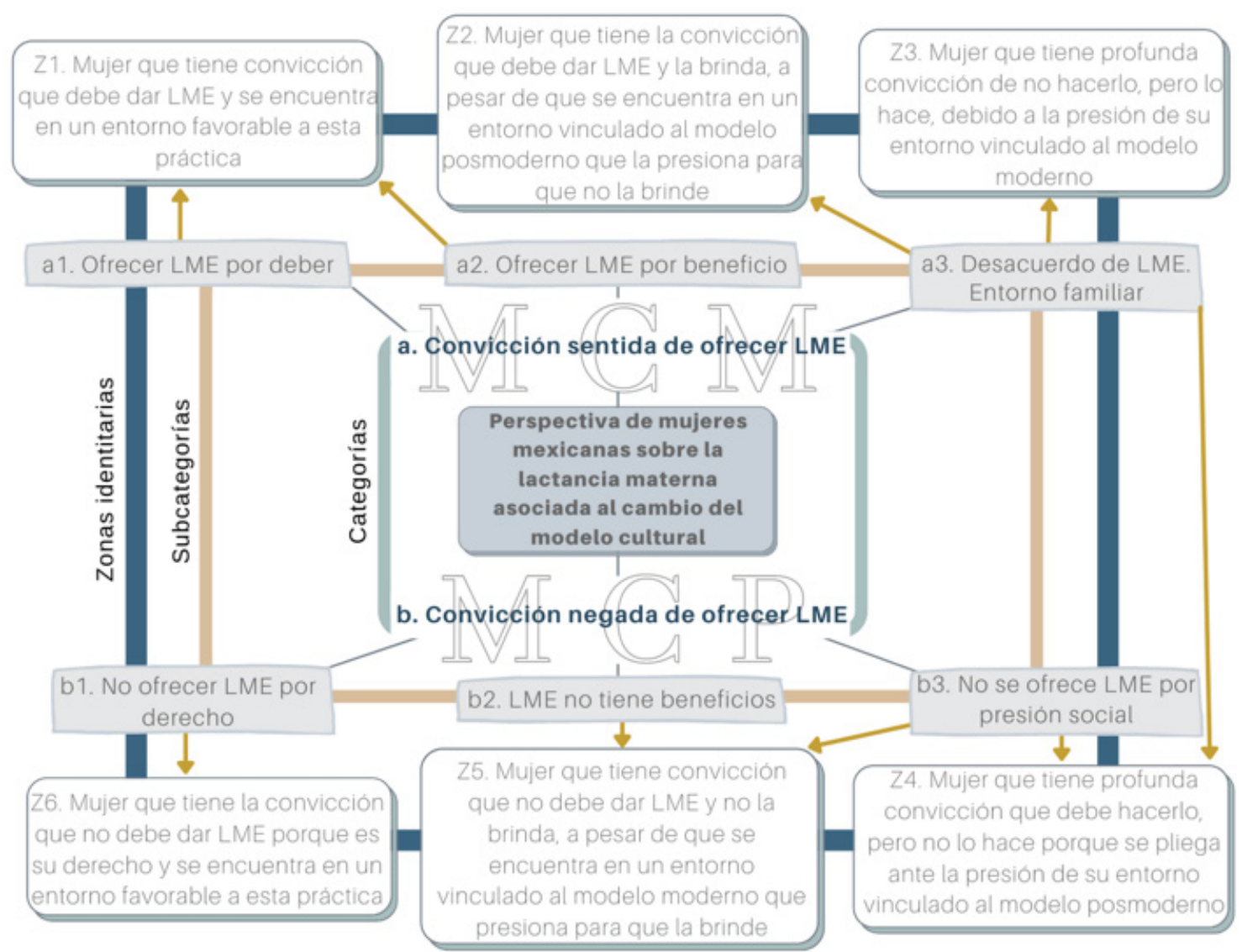

Figura 2. Zonas identitarias de las mujeres según el cambio de modelo cultural

Es decir, son todas aquellas que aun estando en una nueva época de la vida, continúan con las costumbres de sus madres, abuelas, etc., con la finalidad de seguir practicando la LME, por tanto, se encuentran dentro del MCM. 


\section{Categoría b. Convicción negada de ofrecer Lactancia Materna Exclusiva}

La convicción negativa implica una serie de causalidades que orillan a las mujeres a abandonar esta práctica; por ejemplo, las presiones sociales o en el lugar de trabajo, la mercadotecnia, la importancia de la apariencia física y la falta de asesoramiento por parte del personal de salud. Un factor importante que se advirtió durante las entrevistas es la presencia de la convicción negativa de la madre por asumir esta práctica, es decir, no quiere ofrecerla (Figura 3).

Desde que me alivie pensé en no dar pecho, me dolían horrible los senos, y pensé, si no quiero dar leche pues es mi derecho no darla y si mi familia me apoya y mi bebé está bien pues no le doy, si hay leche en polvo por qué voy a sufrir (ríe). [Angélica, 33 años]

- Considero que es nuestro derecho de querer dar o no, sin tener críticas de la sociedad y que no te obliguen, porque al final ni disfrutas darle pecho a tu

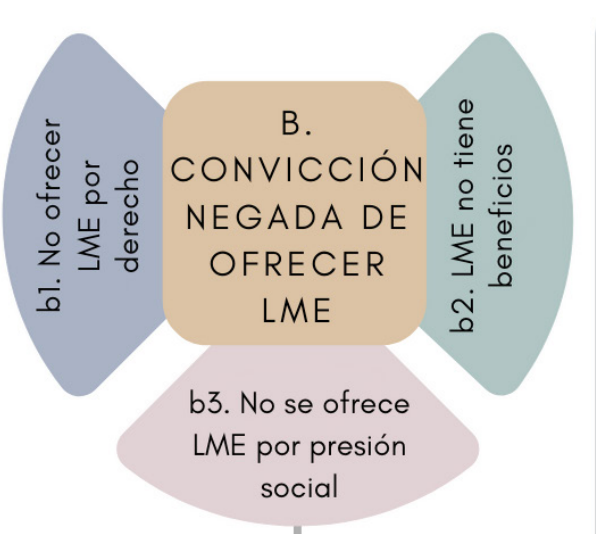

- Yo no quise darle porque se me hace más fácil preparar la fórmula, es más rápido, además en la tele dicen que son buenas y trae buenos nutrientes para mi bebé. [Ana, 25 años]

- Yo no digo que la leche de uno sea mala, pero creo que la leche en polvo es buena porque trae muchas vitaminas, sino no la venderían (ríe). [Monse, 22 años]

- Le empecé a dar fórmula porque mi leche era pura

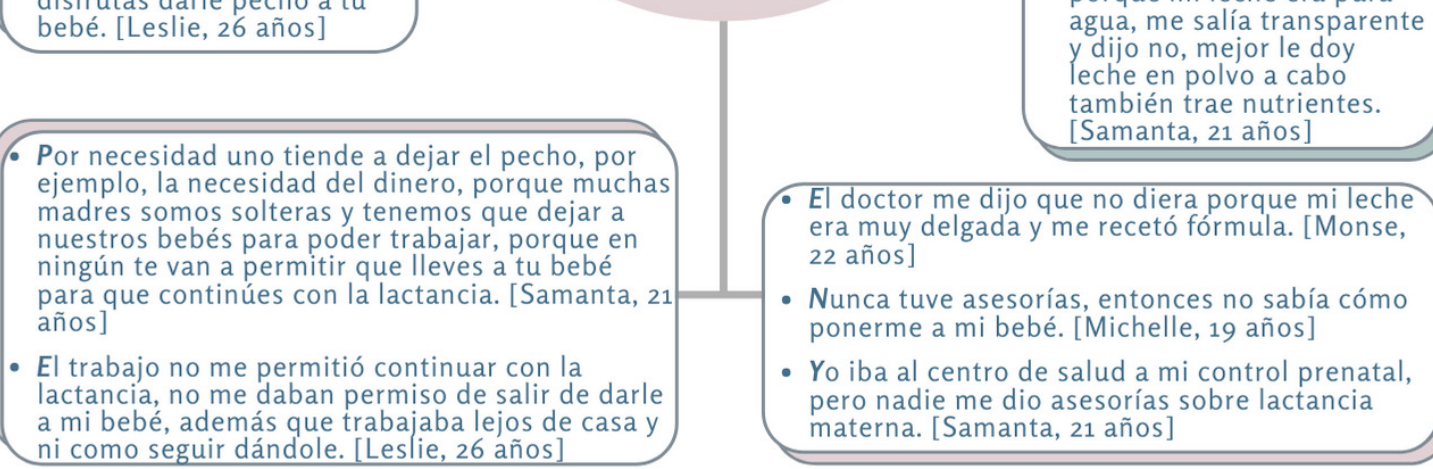
bebé. [Leslie, 26 años] agua, me salía transparente y dijo no, mejor le doy leche en polvo a cabo también trae nutrientes. [Samanta, 21 años] ejemplo, la necesidad del dinero, porque muchas madres somos solteras y tenemos que dejar a nuestros bebés para poder trabajar, porque en ningún te van a permitir que lleves a tu bebé para que continúes con la lactancia. [Samanta, 2 años]

- El trabajo no me permitió continuar con la lactancia, no me daban permiso de salir de darle a mi bebé, además que trabajaba lejos de casa y ni como seguir dándole. [Leslie, 26 años]

\section{Figura 3. Esquematización sobre la opinión respecto a la categoría $b$. Convicción negada de ofrecer LME}

Subcategoría b1. No ofrecer LME por derecho. El derecho es caracterizado por la posición de una persona. Actualmente las mujeres tienen una visión diferente sobre el tema de LM y plantean la decisión de no ejercerla, la madre decide abandonar totalmente la crianza del bebé con su leche por considerarlo un derecho.

Subcategoría b2. LME no tiene beneficios. Hoy en día el fortalecimiento en la industria de los sucedáneos se encuentra supeditado entre el incremento vertiginoso del consumo y altas ventas registradas, lo que impacta directamente en la disminución de las prácticas de LM, al considerar que las fórmulas u otros productos comerciales son mejores que la leche materna.

Subcategoría b3. No se ofrece LME por presión social. El porcentaje de madres que inician la LME ha sufrido fluctuaciones debido a la falta de uniformidad sobre la información recibida por parte de los profesionales sanitarios, la incorporación al mercado laboral, así como no contar con apoyo de la familia. Estas últimas son algunas situaciones que han encaminado a las mujeres de México a tener un fracaso en la continuidad de la lactancia. 
Con relación al modelo cultural estas mujeres se ubican en la era, es decir, en un nuevo modelo. Las razones que sustentan esta idea son: el consumismo; poseen nuevas ideas, costumbres y pensamientos; tienen otras prioridades antes que ofrecer LME al bebé. Los obstáculos presentados en dichas características identitarias son: las dudas, la indecisión, la imposibilidad de creer o elegir entre sus expectativas contradictorias de lo que ellas quieren y lo que su entorno espera. Por tanto, en la categoría bi, las mujeres que no ofrecen LME no generan contradicción, en este grupo la familia apoya su decisión. En consecuencia, este grupo de mujeres se les asigna en las siguientes zonas identitarias:

4. Mujer que tiene profunda convicción que debe hacerlo, pero no lo hace porque se pliega ante la presión de su entorno vinculado al modelo posmoderno, 5. Mujer que tiene convicción que no debe dar LME y no la brinda, a pesar de que se encuentra en un entorno vinculado al modelo moderno que presiona para que la brinde y 6. Mujer que tiene la convicción que no debe dar LME porque es su derecho y se encuentra en un entorno favorable a esta práctica. Conforme a lo anterior, se puede sustentar que se encuentran dentro del MCP (Figura 2).

De este modo las categorías emergidas por los discursos pueden probar que existen diferentes zonas identitarias en mujeres de nuestro país e impactan de manera positiva o negativa para continuar con el amamantamiento.

\section{DISCUSIÓN}

Al analizar los datos se observa que las mujeres se encuentran en dos situaciones, a unas les ayuda a continuar con la lactancia o abandonarla.

Para aquellas mujeres que decidieron por convicción propia ofrecer LME realizan la práctica por el deber de dar este alimento, conocen los beneficios y cuentan con el apoyo de su familia. Esto coincide con Castillas ${ }^{25} \mathrm{y}$ Afoakwah ${ }^{26}$ quienes encontraron que las mujeres sienten la responsabilidad de ofrecer LME, ya que es concebida como parte del sistema de cuidados esenciales durante los primeros meses de vida del bebé.

Por otra parte, distintas investigaciones ${ }^{27,28}$ reportaron que los beneficios de la LME en las madres fueron el vínculo madre-hijo, la protección que proporciona tanto a la madre como al bebé contra enfermedades respiratorias y digestivas, además de defensa y nutrición, además del ahorro económico. Dichos resultados concuerdan con nuestra investigación, las informantes aludieron a los beneficios de la LM como son el apego, la protección y la nutrición. Sin embargo, respecto al ahorro económico, las mujeres de nuestro estudio no lo consideraron como un beneficio.

Durante la revisión de la literatura se encontró que la familia puede ser una fuente importante de apoyo en la instauración inicial de la $\mathrm{LM}^{29,30}$, o por el contrario, puede llegar a influir negativamente en la capacidad y confianza de la madre cuando sus experiencias han sido negativas durante la lactancia ${ }^{31,32}$. En este estudio el apoyo de las parejas parece influir en mayor medida en las madres, por lo que sería conveniente tener presente dichos aspectos y continuar investigando al respecto, así como diferenciar el papel que puede tener cada uno.

En cuanto a la categoría $b$, sobre el derecho de no ofrecer LM, Calvo ${ }^{33}$, Díaz ${ }^{27}$ y Alfonso ${ }^{34}$, han descrito que cuando la madre lleva a cabo el destete al considerarlo un derecho, perciben mayor aceptación por la sociedad, advierten el deterioro de la apariencia de las mamas y la vergüenza de amamantar a sus hijos en público, otras consideran que es más elegante la alimentación con el biberón y que este es un símbolo de estatus social. 
Estudios realizados en España 35,36 encontraron que el contacto de la madre con personal de salud durante el control prenatal y posparto está asociado con la duración de la LME. En la presente investigación el personal de salud es un influyente negativo para que las mujeres no amamanten, ya que las informantes mencionan que les recomiendan las fórmulas, específicamente el médico, así como la falta de asesoría por parte de enfermería.

A pesar de obtener hallazgos destacados, el hecho de que este estudio sea uno de los primeros esfuerzos por lograr un mayor entendimiento respecto a las principales causas del abandono de la LME asociadas al CMC por el que atraviesa la mujer mexicana, y las características de mujeres encontradas, deja abiertas muchas interrogantes como esclarecer las razones por las cuales la mujer se orilla a abandonar esta práctica a pesar del conocimiento de los beneficios que contiene el ofrecerla y lo más importante, qué hacer para coadyuvar el aumento de esta práctica como profesionales de enfermería.

\section{CONCLUSIONES}

La LME aporta grandes beneficios no solo a los bebés sino también a las madres, y especialmente a la sociedad, por ello es necesario que esta práctica se promueva en nuestro medio; pues la tasa baja de LM en México representa un problema para la salud pública. Para lograr esto es necesario conocer los modelos culturales (moderno y posmoderno), además de las características identitarias de mujeres con el fin de comprender cómo es el comportamiento de estas en los diferentes modelos.

Al asociar la LME con los modelos culturales se constata que en el modelo moderno se relaciona con mujeres que ofrecen LME por convicción propia y se considera a este tipo de práctica como un deber; mientras que en el modelo posmoderno las mujeres no ofrecen LME, decisión fundada en un derecho. Al encontrarnos en el tránsito de un modelo hacia el otro, las mujeres están influenciadas por discursos y prácticas culturales que en ocasiones resultan contradictorias e inclusive en algunos momentos, los modelos terminan convirtiéndose en híbridos.

La maternidad y la lactancia materna suponen un proceso de cambio que genera necesidades, dependen tanto de las características socioculturales de los progenitores, como del propio sistema familiar y social. Con este estudio se muestra la gran influencia que tiene la presión social en las mujeres con relación a la elección de la lactancia materna y de los factores que contribuyen a su mantenimiento asociados a las creencias, la cultura y el entorno familiar. El apoyo familiar se considera un determinante tanto en la elección de la LME, como en su continuidad.

Nuestros hallazgos confirman que las mujeres mexicanas atraviesan por un CMC, situación que afecta su vida física, sentimental, familiar, social, laboral y financiera, es decir, todas las esferas de la vida. Asimismo, algunos aspectos importantes que surgieron en la presente investigación fueron el aumento del uso de las fórmulas artificiales, la mercadotecnia y nuevos paradigmas maternos que de manera negativa influyen en la decadencia de la LME.

La población estudiada se obtuvo de un centro de estimulación temprana donde asistían una diversidad de mujeres con diferentes estatus sociales, lo que robusteció los hallazgos y la diferenciación de las zonas identitarias de las mujeres dentro de los cambios de modelos culturales. No obstante, estos hallazgos pueden variar en otro tipo de población.

Falta desarrollar las razones culturales que presenta cada tipo de mujer de acuerdo al estatus económico en el que se encuentra, así como más líneas de investigación relacionados a este tema.

Dicho estudio ha sido elaborado para tratar de develar otras causas que reorienten las políticas y estrategias educativas, ya que la forma en la que se está enfrentando las tasas bajas de la LME no 
han sido del todo efectivas según porcentajes publicados. De igual forma, dar a conocer relatos de las mujeres mexicanas puede contribuir a que la enfermería promocione la LME al elaborar estrategias, investigaciones y políticas públicas con el fin de impulsar el incremento de la LME a nivel nacional.

\section{RESPONSABILIDADES ÉTICAS}

Protección de personas y animales. Los autores declaran que en esta investigación no se han realizado experimentos en seres humanos ni en animales.

Confidencialidad de los datos. Los autores declaran que en el presente artículo no se evidencian datos que permitan identificar a los pacientes.

Derecho a la privacidad y consentimiento informado. La investigación se sujetó a lo establecido en la declaración de Helsinki, se aplicaron los principios bioéticos de no maleficencia, beneficencia, justicia y autonomía.

Financiamiento. Ninguno.

Conflicto de interés. Ninguno.

Agradecimientos. Al grupo de informantes que colaboraron para dicha investigación.

\section{REFERENCIAS}

1. Organización Mundial de la Salud / Organización Panamericana de la Salud. Sesión 1: La importancia de la alimentación del lactante y del niño pequeño y las prácticas recomendadas. En OMS/OPS. La alimentación del lactante y del niño pequeño: Capítulo Modelo para libros de texto dirigidos a estudiantes de medicina y otras ciencias de la salud. Washington, D.C.: OPS: 2010.pp. 3-8 https://bit.ly/33BnMKJ

2. Quispe-Ilanzo MP, Oyola-García AE, Navarro-Cancino M, Silva-Mancilla JA. Características y creencias maternas asociadas al abandono de la lactancia materna exclusiva. RCSP. 2015; 41(4): 582-92.

https://bit.ly/2NoxH3p

3. Secretaría de Salud. Estrategia Nacional de Lactancia Materna 2014-2018. México, D.F.: Secretaría de Salud. https://bit.ly/2Y4IRue

4. González de Cosío-Martínez T, Hernández-Cordero S. Lactancia materna en México. México, DF: Academia Nacional de Medicina de México; 2016. https://bit.ly/3k5pvhr

5. Argomedo AL, Bribiesca-Godoy F, Espinosa-Aguilera A. Reyes-Vázquez H. CAALMA Curso Avanzado de Apoyo a la Lactancia Materna. México, Distrito Federal: APROLAM; 2008. http://bit.ly/2fmMN4U

6. Veneman AM. Retomando el tema de la lactancia materna en la región de América Latina y el Caribe, un compromiso de UNICEF. Panamá: UNICEF; 2009.

7. Instituto Nacional de Salud Pública / UNICEF México. Encuesta Nacional de Niños, Niñas y Mujeres 2015 - Encuesta de Indicadores Múltiples por Conglomerados 2015, Informe Final. México: Instituto Nacional de Salud Pública-UNICEF; 2017. https://uni.cf/35O4seJ

8. Gutiérrez JP, Rivera-Dommarco J, Shamah-Levy T, Villalpando-Hernández S, Franco A, Cuevas-Nasu L, et al. Encuesta Nacional de Salud y Nutrición 2012, Resultados Nacionales. Cuernavaca, México: Instituto Nacional de Salud Pública; 2012. https://bit.ly/3cen5tA

9. González de Cosío-Martínez T, Hernández-Cordero S, Rivera-Dommarco J, Hernández-Ávila M. Recomendaciones para una política nacional de promoción de la lactancia materna en México: postura de la Academia Nacional de Medicina. Salud pública Méx. 2017; 59(1): 106-13. https://doi.org/10.21149/8102 
10. Bajoit G. El cambio sociocultural. México: Universidad Nacional Autónoma de México, Instituto de Investigaciones Sociales; 2010. http://bit.ly/229YBWz

11. Sandoval M. El trabajo social chileno en un contexto de mutación cultural: Desafíos para la acción y la formación. Perspect., Notas interv. acción social. 1999; (7): 3-17.

https://doi.org/10.29344/07171714.7.1153

12. Xavier-Amara LJ, Dos Santos-Sales S, Carvalho-de Souza Rego Pinto DP, Pereira-Cruz GK, Campos-de Azevedo I, Ferreira-Júnior MA. Factors that influence the interruption of exclusive breastfeeding in nursing mothers. Rev. Gaúcha Enferm. 2015; $36($ spe): 127-34.

http://dx.doi.org/10.1590/1983-1447.2015.esp.56676

13. Estévez-González MD, Martell-Cebrián D, Medina-Santana R, García-Villanuev E, Saavedra-Santana P. Factores relacionados con el abandono de la lactancia materna. An Pediatr. 2002; 56(2): 144-50. https://doi.org/10.1016/S1695-4033(02)78944-3

14. Flores-Díaz AL, Bustos-Valdés MV, González-Solís R, Mendoza-Sánchez HF. Factores relacionados con la duración de la lactancia materna en un grupo de niños mexicanos. Arch. Med. Fam. 2006; 8(1): 33-39. http://bit.ly/1XhYeZi

15. Delgado-Becerra A, Arroyo-Cabrales LM, Díaz-García MA, Quezada-Salazar CA. Prevalencia y causas de abandono de lactancia materna en el alojamiento conjunto de una institución de tercer nivel de atención. Bol. Med. Hosp. Infant. Mex. 2006; 63(1): 31-9. http://bit.ly/21EQalB

16. De Souza-Minayo MC. La artesanía de la investigación cualitativa. Buenos Aires: Lugar Editorial; 2009

17. Hernández-Sampieri R, Baptista-Lucio P, Fernández-Collado C, Baptista P. Metodología de la investigación. $4^{\text {ta }}$ ed. México: McGraw-Hill Interamericana; 2006.

18. De Souza-Minayo MC, Ferreira-Deslandes S, Cruz-Neto O, Gomes R. Investigación social: teoría, método y creatividad. Buenos Aires, Argentina: Lugar Editorial; 2003.

19. Secretaría de Salud. Título segundo: De los aspectos éticos de la investigación en seres humanos. En: Constitución Política de los Estados Unidos Mexicanos. Reglamento de la Ley General de Salud en Materia de Investigación para la Salud. México: Diario Oficial de la Federación; 1984. Artículos 2, 13, 14,17, 18. http://bit.ly/1SBpqPT

20. Bioeticaweb. c2004. Comisión Nacional para la Protección de los Sujetos Humanos de Investigación Biomédica y del Comportamiento. Informe Belmont:. Principios y guías éticos para la protección de los sujetos humanos de investigación. https://bit.ly/zdouRYp

21. Mazzanti-Di Ruggiero. Declaración de Helsinki, principios y valores bioéticos en juego en la investigación médica con seres humanos. Rev. colomb. bioet. 2011; 6(1): 125-44.

https://bit.ly/3lcXLY8

22. Guba E, Lincoln YS. Effective evaluation: improving the usefulness of evaluation results through responsive and naturalistic approaches. San Francisco: Jossey-Bass Publishers; 1981.

23. Bajoit G. La tiranía del “Gran ISA". Cultura representaciones soc. 2009; 3(6): 9-24. https://bit.ly/znmZfRm

24. Suárez HJ. La sociología en una sociedad en mutación: entrevista con Guy Bajoit. Argumentos. 2005: (48-49); 191-6. https://bit.ly/3iCGZ2W

25. Castilla MV. La ausencia del amamantamiento en la construcción de la buena maternidad. Rev. estud. género ventana. 2005; 189- 218. https://bit.ly/36yCa8z

26. Afoakwah G, Smyth R, Lavender DL. Women's experiences of breastfeeding: A narrative review of qualitative studies. Afr J Midwifery Womens Health; 2013; 7(2): 71-7.

https://doi.org/10.12968/ajmw.2013.7.2.71 
27. Díaz-Colina JA, Díaz-Colina M, Fernández-Martínez L, Sarría J. Educación prenatal y lactancia materna. Mediciego. 2006; 12(1).

28. Correa-Suárez GM. Significado de la experiencia de la lactancia materna exclusiva para la madre en los primeros seis meses de vida de su hijo. [Tesis]. Bogotá, Colombia: Universidad Nacional de Colombia, Facultad de Enfermería; 2014. https://bit.ly/2GwaLtd

29. Aguilar-Palafox MI, Fernández-Ortega MA. Lactancia materna exclusiva. Rev. Fac. Med. 2007; 50(4): 174-8. https://bit.ly/3jH6yBi

30. Gail CC, Krell JK. Changing the breastfeeding conversation and our culture. Breastfeed Med. 2014; 9(2): 53-5. https://doi.org/10.1089/bfm.2014.9994

31. Jiménez-Sesma ML, Pardo-Vicastillo V, Ibánez-Fleta E. La influencia del profesional sanitario en la lactancia materna. Med. natur. 2009; 3(2): 77-85. https://bit.ly/30HaeLM

32. Schanler RJ, Potak DC. Breastfeeding: Parental education and support. UpToDate; c2O11. https://bit.ly/2FaR7lY

33. Calvo-Quirós C. Factores socioeconómicos, culturales y asociados al sistema de salud que influyen en el amamantamiento. Revenf. 2008; (15). https://bit.ly/3dbvM8n

34. Alfonso-Herrero L, Gorrita-Pérez RR. ¿Cómo influyen diferentes factores psicosociales en el abandono precoz de la lactancia materna? Medimay. 2009; 15(3): 28-34. http://bit.ly/23Dbyay

35. Rius JM, Ortuño J, Rivas C, Maravall M, Calzado MA, López A, et al. Factores asociados al abandono precoz de la lactancia materna en una región del este de España. An Pediatr (Barc). 2014; 80(1): 6-15. https://doi.org/10.1016/j.anpedi.2013.05.011

36. Gil-Estevan MD, Solano-Ruíz MC. Diversidad cultural y lactancia materna. Prestación de cuidados culturalmente competentes en Atención Primaria. Index enferm. 2017; 26(3):162-5. https://bit.ly/znqRTMW 